Ann. Biol. anim. Bioch. Biophys., 1972, 12 (2), 329-334.

\title{
DOSAGE RAPIDE OXYGRAPHIQUE DU GLUCOSE EN PRESENCE DE GLUCOSE-OXYDASE DANS LES MILIEUX COMPLEXES ( $\left.{ }^{1}\right)$
}

\author{
M. JEMMALI et R. KABANA \\ Service des mycotoxines de la Station de Biochimie \\ et Physico-Chimie des Céréales, I. N. R. A., \\ 16, Rue Nicolas Fortin, Paris - $13 \mathrm{e}$ \\ Department of Botany and Microbiologie, \\ Auburn University, Alabama \\ RÉSUMÉ
}

Une technique de dosage très rapide du glucose au moyen d'un oxygraphe et en présence de glucose-oxydase est mise au point. La méthode est susceptible de s'appliquer dans un domaine de concentrations en glucose très étendu. Elle présente de nombreux avantages sur la méthode enzymatique. A titre d'exemple, on applique cette technique au dosage du glucose résiduel dans des préparations colorées de prémélanoïdines.

\section{INTRODUCTION}

Si le dosage du glucose seul ne présente aucune difficulté, sa détermination en milieu complexe demande, par contre, quelques précautions. En effet, certains composés présents dans le milieu sont susceptibles d'avoir des propriétés chimiques analogues à celles sur lesquelles sont fondés les principes des méthodes usuelles de dosage du glucose. C'est pourquoi il convient de le doser :

- soit après l'avoir séparé de l'ensemble du mélange ;

- soit en appliquant directement au mélange la méthode enzymatique initialement proposée par HuGGET et Nixon (I957).

Jemmali et Petir (rg66) ont montré que les résultats obtenus dans le dosage du glucose en présence de produits de la réaction de Maillard, par voie chimique après séparation et par voie enzymatique, concordent.

(1) Une publication sur le même sujet, par les mêmes auteurs, est parue en r97o dans Analytical Biochemistry, 37 (2). 
Compte tenu du développement de l'enzymologie, le dosage manuel du glucose par la méthode enzymatique reste la méthode de choix en raison de sa spécificité et de sa relative rapidité. Cependant, cette méthode, telle qu'elle est décrite par Huggetr et Nixon, et modifiée depuis par Fleming et PEgler (I963), puis par LLOYD et WHELAN (I969), présente certains inconvénients :

- elle a un domaine d'application limité (la meilleure sensibilité se situant entre 5 et $80 \mu \mathrm{g})$;

- elle est coûteuse (utilisation de 2 enzymes et d'un chromogène : l'orthodianisidine;

- elle exige, pour 1'obtention d'une bonne reproductibilité, la mise en ouvre de conditions strictes de température, d'éclairement et de durée pour le développement de la réaction à l'orthodianisidine ;

- elle nécessite, pour sa mise en œuvre, plus d'une heure.

Afin de remédier à ces inconvénients, nous avons entrepris la mise au point d'une méthode de dosage oxygraphique du glucose en présence de glucose oxydase. Dans le présent travail, nous décrivons le principe du dosage, puis nous exposons, a titre d'exemple, les résultats d'analyses du glucose résiduel effectuées dans des préparations de prémélanoïdines obtenues à partir du glucose et de glycocolle. Ces valeurs sont comparées à celles obtenues par JEMMaI, et PETIT (I966) par la méthode enzymatique et par voie chimique après séparation du glucose par chromatographie sur colonne.

\section{MATÉRIEL ET MÉTHODES}

\section{I. - Matériel d'étude utilisé}

Appareillage et réactifs utilisés.

- Oxygraphe (1) avec enregistreur et bain-marie, décrit par LESSLER et al. (1956) fig. 1.

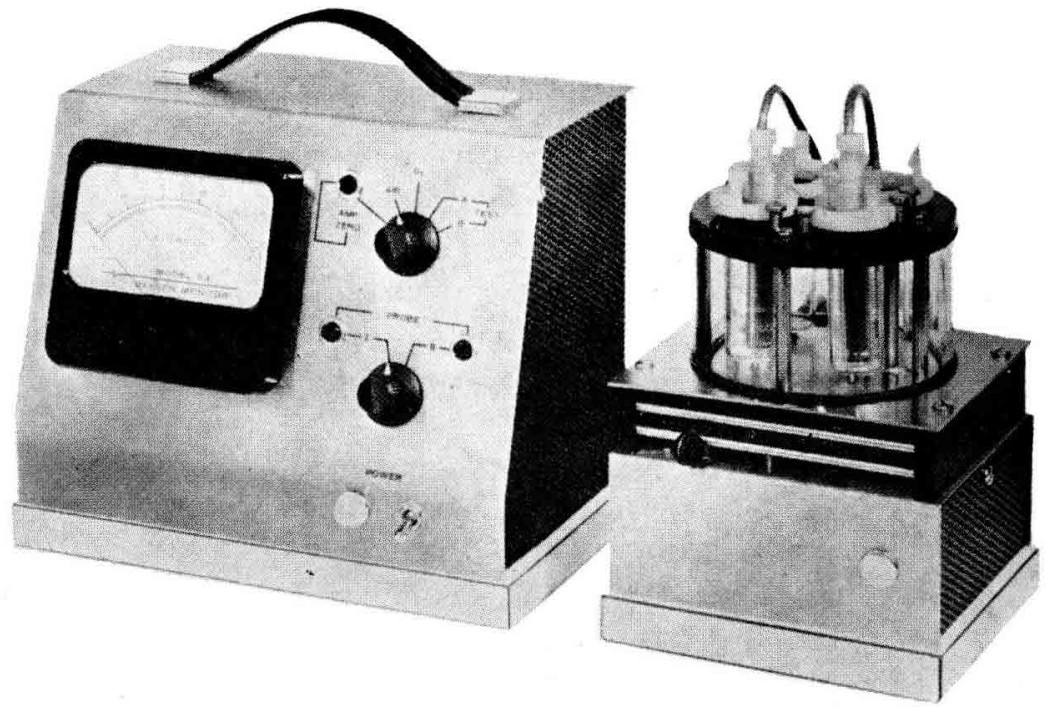

FIG. I. - Oxygraphe Model 53 Biological oxygen, avec bain-marie

(1) Model 53 Biological oxygen Monitor (Yellow Springs Instruments). 
- glucose-oxydase ( $\left.{ }^{1}\right): 30$ ooo unités/g ;

- tampon acétate-glycérol : à une solution de $340 \mathrm{ml}$ de tampon acétate $0,2 \mathrm{M}(\mathrm{pH}=5,6)$, ajouter $660 \mathrm{mll}$ de glycérol ;

- glucose pur $\left({ }^{1}\right)$;

— préparation enzymatique : $30 \mathrm{mg}$ de glucose-oxydase dans $20 \mathrm{ml}$ de tampon acétate glycérol.

$$
\text { 2. - Méthode proposée }
$$

Principe.

Par une réaction d'oxydation catalysée par la glucose-oxydase, le glucose est transformé en acide gluconique avec libération d'eau oxygénée.

L'oxygraphe enregistre automatiquement l'évolution dans le temps de la consommation d'oxygène correspondant à l'oxydation du glucose.

\section{Mode opératoire.}

- Placer I ml de la solution de l'échantillon à analyser (zone de concentration du glucose pouvant varier de 5 à $800 \mu \mathrm{g}$ ) dans la cuve de l'oxygraphe thermostatée à $30^{\circ} \mathrm{C}$.

- Ajouter I $\mathrm{ml}$ de tampon acétate-glycérol.

- Plonger l'électrode dans la cuve jusqu'au niveau supérieur du liquide.

- Mettre l'agitateur magnétique en marche pour homogénéiser la solution.

- Après 3 minutes de mise en température, saturer l'électrode en $\mathrm{O}_{2}$ en réglant l'aiguille de l'oxygraphe au roo $\mathrm{p}$. roo de saturation.

- Ajouter $0,2 \mathrm{ml}$ de la solution enzymatique par la tubulure latérale de la cuve.

\section{Expression des résultats.}

Une durée d'enregistrement d'une minute permet de déterminer la tangente à l'origine des courbes. Un diagramme d'étalonnage est établi en portant les valeurs des tangentes en fonction des concentrations en glucose de o à $800 \mu \mathrm{g}$ par ml de prise d'essai.

\section{RÉSULTATS}

\section{Justification de l'allure de disparition du glucose}

Les résultats relatifs aux valeurs des tangentes correspondant à des concentrations en glucose de o à $800 \mu \mathrm{g}$ par $\mathrm{ml}$ de prise d'essai, sont rassemblés dans le tableau $\mathrm{x}$.

Afin d'éprouver le degré de liaison entre les deux facteurs (concentration initiale en glucose - valeur de la tangente à l'origine de la courbe, nous avons calculé le coefficient $r$ qui est égal à $0,99(\mathrm{P}<0,0 \mathrm{I} ; n=\mathrm{I} 2)$. Il y a donc une liaison hautement significative entre les teneurs en glucose et les vitesses initiales de disparition de celui-ci.

La courbe étalon représentée dans la figure 2 montre que cette liaison est linéaire, ce qui confirme que, dans les conditions expérimentales utilisées, la cinétique d'action de la glucose-oxydase sur le glucose est bien une réaction de premier ordre. L'équation de la droite de régression calculée à partir des données du tableau I correspond à :

$$
y=0,003 x+0,002
$$

$x$ représentant la teneur en glucose et $y$ la valeur de la tangente initiale à la courbe,

(1) N. B. C., Cleveland 28, Ohio. 
ce qui indique, en toute rigueur, une très faible variation de la pression d' $\mathrm{O}_{2}$ pour une extrapolation à une concentration en glucose nulle. Le fait de négliger cette ordonnée à l'origine introduirait une erreur systématique inférieure à 2 p. roo.

\section{TABLEAU I}

Valeur des tangentes à l'origine des courbes de variation de pression d' $\mathrm{O}_{2}$ en fonction des quantités de glucose utilisées (1)

\begin{tabular}{c|c}
\hline $\begin{array}{c}\text { Teneurs en glucose } \\
\text { exprimées en } \mu g\end{array}$ & $\begin{array}{c}\text { Tangente à l'origine } \\
\text { de la courbe }\end{array}$ \\
\hline 0 & 0 \\
5 & 0,015 \\
10 & 0,030 \\
20 & 0,053 \\
30 & 0,089 \\
40 & 0,120 \\
50 & 0,152 \\
80 & 0,242 \\
100 & 0,320 \\
200 & 0,540 \\
400 & 1,230 \\
800 & 2,430 \\
\hline
\end{tabular}

(1) Chaque valeur représente la moyenne de 2 essais consécutifs.

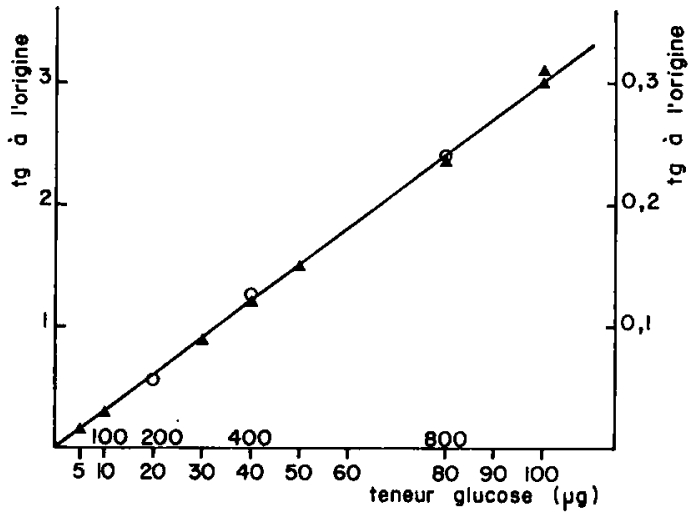

FIG. - 2. L'échelle de gauche est relative aux valeurs en glucose supérieures d $100 \mu$.

(point représentatif : o)

L'échelle de droite correspond à celles comprises entre 0 et $100 \mu \mathrm{g}$.

(point représentatif : + )

\section{Application dans le cas de prémélanoidines}

Les solutions de prémélanoïdines sont préparées selon la méthode décrite par Jemmali et Petit (1966). Les temps de chauffage ont été compris entre 2 et 48 heures. 
Le tableau 2 montre les résultats obtenus sur les différentes préparations de prémélanoïdines avec la méthode chimique de SchaEFFER HARTMANn (I920) après séparation du glucose, donnant une précision de \pm 2 p. Ioo ; la méthode de HUGGET'T et NIxoN et la méthode oxygraphique fournissent une précision de l'ordre de I p. Ioo.

TABLEAU 2

Quantités de glucose résiduel mesurées dans des solutions de prémélanoidines à l'aide de trois différentes méthodes de dosage $\left.{ }^{(}{ }^{1}\right)$

\begin{tabular}{|c|c|c|c|c|c|}
\hline $\begin{array}{c}\text { Temps de chauffage } \\
\text { (en heures) }\end{array}$ & 0 & 2 & 8 & 24 & 48 \\
\hline $\begin{array}{l}\text { Méthode chimique } \\
\text { après séparation }\end{array}$ & 0,40 & 0,39 & 0,35 & 0,26 & 0,17 \\
\hline Méthode enzymatique & 0,40 & 0,39 & 0,33 & 0,27 & 0,17 \\
\hline Méthode oxygraphique & 0,40 & 0,39 & 0,35 & 0,28 & 0,17 \\
\hline
\end{tabular}

(1) Les résultats sont exprimés en millimoles par millilitre de solution utilisée pour les essais.

Chaque valeur représente la moyenne de 5 essais consécutifs.

Le coefficient de variation relatif à la méthode oxygraphique est de 1 p. 100 .

Les données relatives aux deux premières techniques ont été relevées à partir du travail de Jemmali et Pétrit (I966).

Il ressort de ces résultats que, dans la limite des erreurs expérimentales, on retrouve la même concentration en glucose dans les trois cas.

\section{DISCUSSION ET CONCLUSIONS}

Les résultats de la méthode proposée par HuGGETT et NIxoN modifiée peuvent être perturbés :

- soit par intervention de groupements carbonyles des produits de la réaction de Maillard sur $1^{\prime} \mathrm{O}_{2} \mathrm{H}_{2}$ formée au cours de l'oxydation du glucose catalysée par la glucose-oxydase ;

— soit par un phénomène de compétition au niveau du transfert d'hydrogène entre le chromogène ajouté, l'orthodianisidine, et un chromogène susceptible de se former au cours de la réaction de Maillard.

Dans le cas de dosage de glucose dans un milieu complexe très coloré, cette méthode se trouve, par ailleurs, en défaut, en raison de l'interférence de la coloration initiale du milieu avec celle qui se développe en présence d'orthodianisidine. 
La méthode oxygraphique évite ces inconvénients. Elle présente, en outre, les avantages suivants :

- elle est rapide, le dosage demande environ ro minutes ;

- elle est économique, puisqu'elle n'exige l'emploi que d'une seule enzyme;

- elle est susceptible de s'appliquer dans un domaine de concentration en glucose très étendu. Il est même possible de dépasser la valeur de $800 \mu \mathrm{g} / \mathrm{ml}$ à condition de faire en sorte que la réaction enzymatique reste d'ordre $I$; il convient, pour cela d'augmenter la concentration en enzymes, le seul facteur limitant la concentration en glucose étant la capacité de saturation de l'électrode en $\mathrm{O}_{2}$.

Par ailleurs, le réglage de l'appareil (électrode saturée en $\mathrm{O}_{2}$ ) effectué avant l'introduction de la solution de glucose-oxydase permet de compenser toute consommation d'oxygène par d'autres constituants du milieu et assure au dosage une plus grande spécificité. De plus, elle est applicable à la majorité, sinon à la totalité des solutions colorées renfermant du glucose.

Reçu pour publication en novembre 1971.

\section{SUMMARY}

DETERMINATION OF GLUCOSE, IN COMPLEX MEDIA, BY MEANS OF A RAPID POLAROGRAPHIC METHOD WITH GLUCOSE-OXYDASE

A rapid polarographic technique for the determination of glucose with a Clark-type $\mathrm{O}_{2}$ électrode and glucose-oxidase is described. The method is sensitive and accomodates a greater range of glucose concentrations than the conventional spectrophotometric glucose-oxidase procedure. The simplicity and advantage of the polarographic method are discussed. The new technique was tested in the determination of residual glucose in premelanoidins preparations.

\section{RÉFÉRENCES BIBLIOGRAPHIQUES}

Huggett A., St. G., Nixon D. A., r957. Enzymatic determination of blood glucose. Bioch. J., 66, I2 p. Jemmali M., Petit L., ig66. Sur le comportement de deux types de micro-organismes et de différents enzymes en présence de produits de la réaction de Maillard. Ann. Technol agric., :10, 5-42.

Fleming I. D., Pegler H. F., I963. The determination of glucose in the presence of maltose and isomaltose by a stable, specific enzymic reagent. Analyst, 88, 967.

Lloyd J. B., Whelan W. J., I969. An improved method for enzymic determination of glucose in the presence of maltose. Analyst. Bioch., 30, 467.

Lessler M. A., Molloy E., Schamb C. M., 1965. Measurement oxidative activity in biological systems by an automated oxygen electrode. Fed. Proc., 24, 336. 